\title{
SIOP Ependymoma I: Final results, long term follow-up and molecular analysis of the trial cohort: A BIOMECA Consortium Study
}

\author{
Timothy A Ritzmann ${ }^{1,2}$, Rebecca J Chapman ${ }^{1}$, John-Paul Kilday ${ }^{3,4}$, Nicola Thorp ${ }^{5,6}$, Piergiorgio \\ Modena $^{7}$, Robert A Dineen ${ }^{1,2,8}$, Donald Macarthur ${ }^{1,2}$, Conor Mallucci ${ }^{9}$, Timothy Jaspan ${ }^{2}$, Kristian W \\ Pajtler $^{10,11,12}$, Marzia Giagnacovo ${ }^{7}$, Thomas S Jacques ${ }^{13,14}$, Simon ML Paine ${ }^{1,2}$, David W Ellison ${ }^{15}$, Eric \\ Bouffet ${ }^{16}$ and Richard G Grundy ${ }^{1,2}$ on behalf of the BIOMECA consortium
}

(1) Children's Brain Tumour Research Centre, University of Nottingham, UK

(2) Nottingham University Hospitals NHS Trust, UK

(3) Children's Brain Tumour Research Network (CBTRN), Royal Manchester Children's Hospital, UK.

(4) The Centre for Paediatric, Teenage and Young Adult Cancer, University of Manchester, UK

(5) The Clatterbridge Cancer Centre, Liverpool, UK

(6) The Christie Hospital Proton Beam Therapy Centre, Manchester, UK

(7) Genetics Unit, ASST Lariana General Hospital, Italy

(8) NIHR Nottingham Biomedical Research Centre

(9) Alder Hey Children's NHS Foundation Trust

(10) Hopp Children's Cancer Center Heidelberg (KiTZ), Germany

(11) Division of Pediatric Neuro-oncology, German Cancer Research Center (DKFZ) and German Cancer Consortium (DKTK), Germany

(12) Department of Pediatric Oncology, Hematology, and Immunology, University Hospital Heidelberg, Germany

(13) UCL GOS Institute of Child Health, London, UK

(14) Great Ormond Street Hospital for Children NHS Foundation Trust, UK

(15) Department of Pathology, St. Jude Children's Research Hospital, USA

(16) The Hospital for Sick Children, Toronto, Canada

(C) The Author(s) 2022. Published by Oxford University Press on behalf of the Society for Neuro-Oncology.

This is an Open Access article distributed under the terms of the Creative Commons Attribution-NonCommercial License (https://creativecommons.org/licenses/by-nc/4.0/), which permits non-commercial re-use, distribution, and reproduction in any medium, provided the original work is properly cited. For commercial re-use, please contact journals.permissions@oup.com 
Corresponding Dr Timothy Ritzmann and Professor Richard Grundy, University of Nottingham Biodiscovery Institute.Timothy.ritzmann@nhs.net, Richard.grundy@nottingham.ac.uk.

Funding: The study was supported by Cancer Research UK and the CRUK Children's Cancer Trials Team (University of Birmingham). Funding from Fighting Ependymoma, Children with Cancer UK, The Joe Foote Research Foundation, The James Tudor Foundation, Brainstrust and the Associazione Bianca Garavaglia also supported this research. TAR is an NIHR Academic Clinical Lecturer. TSJ received funding from the Brain Tumour Charity, GOSH Children's Charity, Olivia Hodson Cancer Fund, Cancer Research UK. Research at GOSH and UCL Great Ormond Street Institute of Child Health is supported by the NIHR GOSH Biomedical Research Centre.

Conflicts of Interest: The authors have no conflicts of interest.

\section{Authorship:}

Experimental Design: TAR,DM,DWE,EB,RGG

Implementation: TAR,RJC,DM,JPK,CM,NT,PM,TJ,TSJ,SMLP,DWE,EB,RGG

Data Analysis: TAR,RJC,DM,JPK,CM,NT,PM,RD,TJ,TSJ,SMLP,KWP,DWE,EB,MG,RGG

Data Interpretation: TAR,RJC,DM,JPK,CM,NT,PM, RD,TJ,TSJ,SMLP,KWP,DWE,ED, RGG

Manuscript Writing: All

Data Sharing: DNA methylation profiles in this study are deposited in the gene expression omnibus (GSE182707). 


\begin{abstract}
Background: SIOP Ependymoma I was a non-randomised trial assessing event free and overall survival (EFS/OS) of non-metastatic intracranial ependymoma in children aged 3 to 21 years treated with a staged management strategy. A further aim was to assess the response rate (RR) of subtotally resected (STR) ependymoma to vincristine, etoposide and cyclophosphamide (VEC). We report final results with 12-year follow-up and post hoc analyses of recently described biomarkers.
\end{abstract}

Methods: 74 participants were eligible. Children with gross total resection (GTR) received radiotherapy, whilst those with STR received VEC before radiotherapy. DNA methylation, 1q, hTERT, ReLA, Tenascin-C, H3K27me3 and pAKT status were evaluated.

Results: Five- and ten-year EFS was $49.5 \%$ and $46.7 \%$, OS was $69.3 \%$ and $60.5 \%$. GTR was achieved in 33/74 (44.6\%) and associated with improved EFS ( $p=0.003, \mathrm{HR}=2.6,95 \%$ confidence interval (CI) 1.45.1). Grade 3 tumours were associated with worse $\mathrm{OS}(\mathrm{p}=0.005, \mathrm{HR}=2.8,95 \% \mathrm{Cl} 1.3-5.8) .1 \mathrm{q}$ gain and hTERT expression were associated with poorer EFS $(p=0.003, \mathrm{HR}=2.70,95 \% \mathrm{Cl} 1.49-6.10$ and $\mathrm{p}=0.014$, $\mathrm{HR}=5.8,95 \% \mathrm{Cl} 1.2-28)$ and $\mathrm{H} 3 \mathrm{~K} 27 \mathrm{me} 3$ loss with worse $\mathrm{OS}(\mathrm{p}=0.003, \mathrm{HR}=4.6,95 \% \mathrm{Cl} 1.5-13.2)$. Methylation profiles showed expected patterns. 12 participants with STR did not receive chemotherapy; a protocol violation. However, best chemotherapy RR was $65.5 \%$ (19/29, 95\% Cl 45.7 82.1), exceeding the prespecified $45 \%$.

Conclusions: Participants with totally resected ependymoma had the best outcomes. RR of STR to VEC exceeded the pre-specified efficacy criterion. However, cases of inaccurate stratification 
highlighted the need for rapid central review. 1q gain, H3K27me3 loss and $h T E R T$ expression were all associated with poorer survival outcomes.

Keywords: Ependymoma, Chemotherapy, Resection, Recurrence, Radiotherapy. 


\section{Key Points:}

(1) Chemotherapy shows a $65 \%$ response rate in subtotally resected childhood ependymoma.

(2) 1q gain and hTERT associate with poor outcomes in childhood ependymoma.

(3) Prolonged follow-up demonstrates utility of a staged ependymoma management strategy.

\section{Importance of the Study:}

We report original results and twelve-year follow-up of an international paediatric ependymoma clinical trial of 74 participants. Our study reports event free and overall survival of a staged management strategy targeting maximum local tumour control. We demonstrate a measurable response of subtotally resected ependymoma to vincristine, etoposide and cyclophosphamide. We also highlight areas for improvement in trial management which has informed our current Ependymoma II clinical trial. Additionally, we confirm the significance of chromosome 1q gain, hTERT expression and H3K27me3 loss in a trial series and apply DNA methylation profiling. Our study contributes to debate about the efficacy of chemotherapy in childhood ependymoma and shares the long-term outcomes of a previously unreported trial cohort. 


\section{Introduction}

Paediatric ependymomas are associated with poor outcomes ${ }^{1,2}$. Five-year overall survival (OS) above $70 \%$ is rarely reported whilst event free survival (EFS) is around $55 \%{ }^{3-6}$.

Surgical gross total resection (GTR) is associated with improved outcomes ${ }^{7-9}$. Post-operative radiotherapy of (photon) doses up to $59.4 \mathrm{~Gy}$ using $1.8 \mathrm{~Gy}$ per fraction to the tumour bed is now recommended as a standard of care for children over 12 months $^{9}$ and there is increasing use of proton beam radiotherapy. The role of chemotherapy is unclear ${ }^{10}$; some researchers report benefits of chemotherapy, particularly in younger children ${ }^{11-15}$, whilst others report no, or limited, benefits $^{16,17}$.

Significant progress has been made in understanding ependymoma's molecular basis. Posterior Fossa A (PFA) and ZFTA-fusion (formerly RELA-fusion) ${ }^{18}$ supratentorial ependymomas have the worst outcomes $^{19,20}$. Additionally, chromosome 1q gain is a poor prognostic indicator ${ }^{1,3,21-24}$. Other proposed prognostic markers include telomerase activity via $h T E R T^{23,25,26}$, Tenascin-C (TNC) ${ }^{27}$, H3K27me3 loss ${ }^{28,29}$, and pAKT expression ${ }^{30}$.

The Biomarkers of Ependymoma in Childhood and Adolescence (BIOMECA) study is an integral part of the International Society of Paediatric Oncology (SIOP) Ependymoma II trial. Included in BIOMECA's remit is the molecular evaluation of historical clinical trial datasets. Therefore, we present the previously unreported findings of the SIOP Ependymoma I protocol, recruited between 1999 and 2007, with long-term follow up and retrospective analysis of molecular markers. 
The primary aims were:

(1) To determine EFS and OS of participants with ependymoma treated with a staged management strategy targeting maximum local control;

(2) To establish the response rate (RR) of intracranial ependymoma to a combination of vincristine, etoposide and cyclophosphamide (VEC).

\section{Methods}

Eligibility criteria and outcome measures

Eligible patients were 3 to 21 years with untreated non-metastatic, intracranial, histologically confirmed ependymoma. Patients with myxopapillary ependymoma, subependymoma or ependymoblastoma were excluded. Tissue for molecular analysis was not mandated.

Outcome measures were: EFS and OS, surgical operability and RR to chemotherapy.

\section{Trial design (Figure 1A)}

Extent of resection was categorised, according to contemporary standards ${ }^{31,32}$, as either subtotal resection (STR) $\left(>1.5 \mathrm{~cm}^{2}\right.$ residuum on a single cross sectional image) or GTR (no visible residuum, or residuum $<1.5 \mathrm{~cm}^{2}$ ) and determined by radiological consensus, based initially on local opinion then central review. Second-look surgery was recommended for those with operable residuum. 
Participants with GTR underwent focal radiotherapy of 54 Gy in daily fractions of $1.8 \mathrm{~Gy}$ over six weeks.

Participants with STR received up to four cycles of VEC (Figure 1C, Supplementary Material 1) with MRI assessments after cycles two and four. Responses were centrally reviewed according to contemporary recommendations ${ }^{31}$. Percentage response was determined by calculating the product of the perpendicular diameters of the tumour relative to the baseline, post-operative, evaluation:

- Complete Response (CR): no disease;

- Partial Response (PR): 50\% reduction;

- Objective Response (OR): 25-50\% reduction;

- Stable Disease (SD): $<25 \%$ reduction;

- Progressive Disease (PD): $>25 \%$ increase.

Following chemotherapy, the protocol specified that all participants receive focal radiotherapy (Figure 1A). Participants who progressed on treatment were to discontinue chemotherapy and proceed to radiotherapy.

Participants were monitored until censoring or death. The clinicaltrials.gov identifier was NCT00004224. Written informed consent was required and the study was approved by Trent multicentre research ethics committee (MREC99/02/11,CTA MF8000/13710). 
Previous retrospective studies indicated five-year OS for those with primary GTR and STR ranged from 30 to $85 \%$ and 0 to $45 \%$ respectively. As there were no reliable prospective data at the time of study design RR, rather than survival was used to measure chemotherapy efficacy ${ }^{33}$. This study assumed five-year OS of $70 \%$ with GTR and $35 \%$ with STR (HR $=0.34$ favouring GTR). It was assumed that $3 / 8$ would achieve GTR. Using two-sided $5 \%$ significance and $80 \%$ power, a study of 65 participants was proposed (GTR 24, STR 41). On the basis of previously published responses to high-dose chemotherapy combinations in ependymoma ${ }^{34}$, it was anticipated that if RR to chemotherapy in the STR group was under $25 \%$ there would be no interest in the combination. In contrast, RR $45 \%$ or more would suggest worthwhile efficacy and was set as the criterion for chemotherapy response. A one-stage Fleming-A'Hern design required 32 STR participants with a minimum of 13 responses to claim sufficient activity ${ }^{35}$

\section{Analysis}

Data analysis was conducted in R (www.r-project.org). Survival probabilities were calculated using the Kaplan-Meier method. Multivariate analyses used the Cox Proportional Hazards Model through the survival package in R. The influences of tumour resection, location and WHO grade were investigated prospectively. Molecular analyses were post hoc.

EFS was defined as the time from surgery to recurrence, PD or death. OS was defined as time from surgery to death. Surviving participants were censored at date last seen. Best Response was determined as the best response recorded from commencing chemotherapy until progression or chemotherapy completion. RR was calculated as the proportion of CR 
plus PR from all those with STR receiving chemotherapy. 95\% Confidence Intervals (CI) were calculated using the Clopper-Pearson approach ${ }^{36}$.

Due to poor compliance with chemotherapy allocations, an "as-treated" analysis was performed for chemotherapy treated participants to better understand the true RR to VEC.

\section{Molecular Analysis (Supplementary Material 2)}

DNA methylation profiles were generated using Infinium HumanMethylation450 BeadChip arrays (Illumina) (GSE182707). Subgroups were assigned using Heidelberg classifiers v11b4/v12.3 as previously described ${ }^{1} .31 / 34$ cases had calibrated scores $>0.9$ (range $0.84-1$ ).

1q status was evaluated by DNA methylation profiling, fluorescent in-situ hybridisation (FISH) and multiplex ligation-dependent probe amplification (MLPA) as previously described ${ }^{1}$. There was good correlation between methods. The Fleiss-Kappa statistic was $0.615(p<0.001)$ and $0.708(p=0.008)$ where two and three tests were performed respectively.

Quantitative real-time PCR determined $h$ TERT expression as previously described ${ }^{26}$.

Immunohistochemistry was performed in triplicate on four micrometre tissue microarrays for ReLA, TNC, H3K27Me3 and pAKT and were scored as negative or positive (Supplementary Material 2). 


\section{Results}

\section{Cohort Summary}

89 participants with intracranial ependymoma from 25 centres in the United Kingdom, Ireland, Spain, Denmark, Sweden and the Netherlands were registered between $17^{\text {th }}$ May 1999 and $1^{\text {st }}$ November 2007. Four participants had metastatic disease at presentation, whilst 11 did not have a histopathological diagnosis of ependymoma. Of those with a non-ependymoma diagnosis on histopathology, six underwent post hoc DNA methylation profiling, all of which were confirmed as non-ependymoma. No patients were removed on the basis of DNA methylation profile alone. This left 74 participants with non-metastatic intracranial ependymoma (Figure 1B).

Thirty-eight of seventy-four (51\%) participants were male. Median age at diagnosis was 7.8 years (range 3.1-18.8). Forty-seven of seventy-four (64\%) had posterior fossa (PF) tumours and 39/74 (53\%) were WHO Grade 2. Twenty-nine (39\%) participants achieved GTR after first surgery. Of the 45 with initial STR, ten (22\%) had early second-look surgery, four of these (40\%) achieved GTR and six (60\%) still had STR, leaving 41/74 (55.4\%) with STR prior to adjuvant therapy, a GTR rate of 33/74 (44.6\%) (Table 1, Figure 1B).

Surgical Outcomes

Most second-look surgeries (10/16, 63\%) occurred prior to adjuvant therapy, but because decisions on further surgery were taken at local centres, it has not been possible to determine detailed outcomes of second surgery for the six participants undergoing subsequent surgery. To consider second-look surgery in more depth, a surgical panel retrospectively reviewed post-operative scans following STR to independently consider whether they would have attempted further early 
resection. There were limitations to imaging availability, but the surgical panel would have attempted early second resection in $10 / 25$ cases reviewed (40\%). Of these, four actually had early second-look surgery, two of whom achieved GTR.

There was no difference in extent of resection between centres with high numbers of cases (five or more) compared to centres with low numbers (four or fewer) (High volume=18/39 (46.2\%) GTR, Low volume $=15 / 35(42.9 \%) \mathrm{GTR}, \mathrm{p}=0.776$, Chi-square test). There was no difference in age, tumour volume or location between participants with GTR versus STR. Resection rates improved over time; the GTR rate between 1999-2002 was 12/37 (32.4\%), rising to 21/37 (56.8\%) between $2003-2007$ $(p=0.035$, Chi square test). The greatest improvements in resection rates over the trial were in lower volume centres ( $25 \%$ to $50 \%$ ) when compared with higher volume centres ( $43 \%$ to $55 \%$ ).

\section{Survival Outcomes (Table 2)}

Median follow-up for surviving participants was twelve years (range 1.2 to 19). 32/74 (43\%) died: 22/41 (54\%) with STR and 10/33 (30\%) with GTR. 41/74 (55\%) relapsed, 28/41 (66\%) with STR and $13 / 33(40 \%)$ with GTR.

Five- and ten-year EFS was $49.5 \%$ and $46.7 \%$ whilst OS was $69.3 \%$ and $60.5 \%$ (Figures 1D\&E). GTR was associated with improved EFS compared to STR (Five-year EFS $69.1 \%$ Vs $33.8 \%, p=0.003$, $H R=0.38$ ) (Figure 2A). GTR was also associated with a clinically significant improvement of $21.8 \%$ in OS although this did not reach statistical significance (Five-year OS $81.3 \%$ Vs 59.5.0\%, $p=0.071$, $\mathrm{HR}=0.50$ ) (Table 2). Tumour grade was not associated with EFS but WHO grade 3 tumours were associated with worse OS (Five-year OS 52.2\% Vs $84.2 \%, p=0.005, H R=2.8$ ) (Figure 2B). 
On prespecified multivariate analysis of OS including grade, tumour location and extent of resection $(n=74)$, only grade 3 tumours remained a predictor of poorer outcome $(p=0.008, H R=2.73,95 \% C l$ 1.29-5.78). For EFS, only STR predicted worse survival $(p=0.005, H R=2.58,95 \% \mathrm{Cl} 1.33-5.00)$ (Supplementary Material S3).

\section{Treatment outcomes}

In the GTR group ( $n=33), 32$ participants (97\%) received focal radiotherapy. One participant, with supratentorial ependymoma lacking 1q gain, received no further treatment due to family choice and was still alive after ten-years (Figure 1B).

In the STR group ( $n=41)$, despite a clear protocol, 10 participants with residuum proceeded to radiotherapy without chemotherapy (Figure 1B). Of these, four resulted from family preference, and one was because resection was assessed locally as GTR, but STR on subsequent central review. No reason was documented for the remaining five. Two participants received no therapy after STR; one dying within two months of diagnosis and the other progressing after 14 months. 26 STR group participants received chemotherapy followed by focal radiotherapy whilst a further three received chemotherapy alone.

The CR+PR RRs were 14/28 (50.0\%) and 13/22 (59.1\%) after cycles two and four respectively. 19/29 (65.5\%, 95\% Cl 45.7-82.1) participants achieved CR or PR as the best response during chemotherapy, exceeding the prespecified $45 \%$ RR (Figure 3 A). 
Overall, 21/29 participants (72\%) had no progression during chemotherapy. Eight (28\%) had PD by the end of chemotherapy. Six participants had PD after cycle two, of these, two discontinued chemotherapy and four continued. The four who continued chemotherapy despite PD all had local assessments suggesting responses and continued on this basis until central review; by cycle four one of these had CR and three had persisting PD. At cycle four, two participants who had CR at cycle two progressed and one (who missed the cycle two assessment) had PD (Figure 3B).

In a post hoc analysis, the twelve participants in the STR group who did not receive chemotherapy had median OS of 64 months, whilst those who received chemotherapy did not fall below $50 \%$ OS. However, there was no difference between the two groups (OS $p=0.35$, EFS $p=0.56$ ) (Supplementary Material S4).

The main toxicities of chemotherapy were leucopenia (97\%), thrombocytopenia (48\%), nausea and vomiting (24\%), and infection (21\%) (Supplementary Material S5). Two participants, both with CR, stopped chemotherapy after two cycles due to toxicity.

Retrospective molecular analysis of the trial cohort

Thirty-four of seventy-four (46\%) participants had a DNA methylation result and in 60/74 (81\%) 1q status was known. Protein expression was measured in 40/74 (54\%) for TNC, 50/74 (68\%) for pAKT, 54/74 (73\%) for H3K27me3 and 51/74 (69\%) for RelA. 16/74 (22\%) had hTERT expression measured.

Seventeen of thirty-four DNA methylation profiled tumours were PFA (50\%), 10 (29\%) were ZFTAfusion and five (15\%) were PFB. There was one each (3\%) of YAP and neuroepithelial tumour, 
PLAGL1 fusion-positive (ST_PLAGL1) ${ }^{37}$ (Figure 4A). Eighteen of sixty (30\%) had 1q gain (Table 1). Twenty-four of sixty were assessed by three methods (FISH, MLPA and methylation array), 16 with two methods and 20 with one method. 1q gain was identified in 8/17 (47\%) of PFA and 3/10 (30\%) ZFTA-fusion. 1q gain was not seen in PFB or YAP. There was no difference in 1q gain between GTR and STR cases $(p=0.611) . h T E R T$ was only expressed in PFA and ZFTA-fusion tumours. RELA was expressed in PF and supratentorial subtypes. In PF tumours, H3K27Me3 was only expressed in PFB. pAKT positivity was seen in all subtypes whilst TNC expression was restricted to PFA (Figure 4B).

$1 q$ gain was associated with poorer EFS (Five-year EFS 33.3\% Vs 59.0\%, $p=0.003, H R=2.71$ ) and OS (Five-year OS 55.6\% Vs 75.5\%, p=0.042, HR=2.22) (Figures 2C\&D). When further stratified by tumour location, 1q gain was only prognostic in PF tumours (five-year EFS 33.3\% Vs 70.8\%, p=0.002, HR=3.94 and OS 58.3\% Vs 75\%, p=0.023, HR=3.07) ( $n=36)$. No difference in survival was seen for supratentorial tumours in relation to 1q gain $(n=24)$ (Supplementary Material S6).

Consistent with its association with loss of expression in PFA, H3K27me3 positivity was associated with better OS (Five year OS $46.9 \%$ Vs $90.2 \%, p=0.003, H R=0.22$ ) but no significant difference in $E F S$ $(p=0.200)$ (Figure 2E). hTERT was associated with worse EFS (Five-year EFS 20.0\% Vs $83.3 \%, p=0.014$, $H R=5.8$ ) but not OS ( $p=0.092$ ) (Figure 2F). RELA protein, TNC and pAKT expression were not associated with outcome. DNA Methylation classification was not associated with outcome, possibly due to small numbers of cases, but patterns of survival were consistent with previous reports ${ }^{1,20}$ (Figures 2G\&H, Table 2).

On post hoc multivariate analysis, adding $1 q$ status to the prespecified analysis $(n=60)$, only grade 3 tumours remained a predictor of poorer OS ( $p=0.019, \mathrm{HR}=2.82,95 \% \mathrm{Cl} 1.19-6.69) .1 q$ status and STR 
were significant predictors of poorer EFS (1q: $p=0.007, H R=2.78,95 \% C l$ 1.33-5.82, STR: $p=0.004$, $\mathrm{HR}=2.93,95 \% \mathrm{Cl} 1.40-6.15)$ (Supplementary Material S3). Other molecular markers had insufficient cases to include.

\section{Discussion}

Whilst knowledge of ependymoma has advanced since trial design ${ }^{18-20,37-40}$, development of effective treatments has shown little progress ${ }^{1}$. We report long-term outcomes for children treated with a staged management strategy and that the best chemotherapy RR of $65.5 \%$ exceeded a prespecified $45 \%$ rate, supporting the use of VEC for inoperable childhood ependymoma. We also retrospectively applied molecular diagnostics to a clinical trial cohort ${ }^{3,22,26}$.

A primary aim was to assess the role of chemotherapy in inducing a tumour response for participants with STR. The best RR of $65.5 \%$ suggests that VEC is associated with tumour response in this group. Whilst the number receiving chemotherapy was 29 rather than the targeted 32 , the 19 responses exceeded the 13 prespecified for demonstration of efficacy. We assessed RR rather than survival as an outcome because no prospective survival data was available when the study was designed $^{33}$. Further work is required to establish whether chemotherapy provides either a direct survival benefit or an indirect benefit through the facilitation of additional surgery. The post hoc comparison between STR participants with and without chemotherapy was not designed to answer this.

In 12 participants for whom STR was achieved, no chemotherapy was given, in breach of the protocol, additionally four patients with PD continued on chemotherapy when they should have 
discontinued. Both of these inaccuracies resulted from local treatment decisions, as prospective central radiological review was not mandated. Additionally, there were four cases in which the family did not consent to chemotherapy. The challenge of protocol compliance for post-operative chemotherapy regimens in ependymoma is not a unique experience; a similar problem has been reported in $\mathrm{ACNSO831} 1^{41}$ and the SIOP ependymoma II study is experiencing related difficulties (Personal Communication, R Grundy, 2021). We have now included rapid central review within the current SIOP Ependymoma II study and based on this experience, we recommend the use of multidisciplinary meetings at trial registration to enhance protocol compliance.

Whilst a number of studies have identified a role for chemotherapy in children under three years, aiming to avoid or delay radiotherapy ${ }^{12,13,15,22}$, others have disagreed ${ }^{4,6,14}$. Our study demonstrated chemotherapy efficacy in older age groups. This is consistent with the findings of more recent US studies $^{6,42}$. Garvin used a combination of vincristine, cisplatin and etoposide and reported a RR of $57 \%$ in 35 evaluable participants, which is close to our RR of $65.5 \%{ }^{6}$. Massimino reported a cohort of 160 children using VEC chemotherapy to bridge the gap to second resection but did not directly report on the RR ${ }^{4}$. More recently, ACNS0121 investigated children with STR given vincristine, carboplatin, cyclophosphamide and oral etoposide, followed by radiotherapy and second-look surgery with combined CR and PR of $67 \%{ }^{42}$. Preliminary results from the ACNS0831 study indicate a role for chemotherapy in some patients with totally, or near totally, resected ependymoma; maintenance with vincristine, cisplatin, cyclophosphamide and etoposide following radiotherapy was associated with a significantly better 3 -year EFS (80\% versus $71 \%)$. Whilst this chemotherapy was delivered at a different stage of treatment and different resection status to our study, it provides further evidence of a role for chemotherapy in some patients with ependymoma ${ }^{41}$. The results of the STR and chemotherapy arm of ACNS0831 are awaited. The VEC chemotherapy regimen in our trial did not include a platinum based drug. Given the evidence of response of tumour residuum to VEC, 
future studies must consider the benefits of platinum chemotherapy against the risk of nephrotoxicity and ototoxicity.

Although not a primary aim, we have attempted to answer important questions related to surgery. The importance of GTR in obtaining good outcomes has been re-emphasised in this study, with improved EFS. It is important to note that our definition of GTR was less stringent than the present day. In line with standards at the time of protocol design, GTR was accepted as $<1.5 \mathrm{~cm}^{2}$ tumour on cross sectional imaging. In the present SIOP Ependymoma II study, $1.5 \mathrm{~cm}^{2}$ of tumour would be classified as residuum $\mathrm{R} 3$ making resection rates difficult to directly compare. A retrospective review of scans attempted to elucidate factors behind incomplete surgery. This was hampered by limited availability of complete sets of pre- and postoperative scans. However, as reported, no differences between GTR or STR participants were identified.

This study was powered for a $38 \%$ GTR rate and bettered this with a rate of $45 \%$. It is notable that GTR rates rose from $32.4 \%$ to $56.8 \%$ during the trial. Surgeons have become increasingly aware of the prognostic impact of GTR on ependymoma outcomes and, in association with this, resection rates reported in clinical trials have increased. However, surgeons are frequently not aware of the diagnosis at surgery. Review of a subgroup of operation notes in this study revealed that smear or frozen section results were frequently non-committal or incorrect. In less than half of cases in which operation notes were reviewed was there either confident suggestion of ependymoma or confirmed histology. Better pre-operative diagnosis may aid surgical planning and improve resection rates. Improved imaging techniques may help to address this challenge ${ }^{43}$. 
The small number of participants undergoing further early resection makes it hard to assess the added value of attempting to convert STR to GTR with repeated surgery. The retrospective surgical panel felt that twice as many participants may have been suitable for second-look surgery as occurred ( $40 \%$ versus $22 \%$ ). As a result of this experience, earlier consideration of further surgery by an independent panel alongside close assessment of the morbidity associated with repeat surgery is a focus of the SIOP Ependymoma II trial ${ }^{44}$. A weekly panel review aims to deliver more definitive answers on the benefits and morbidity of exhaustively pursuing GTR. This is critical, as surgery for cerebellopontine angle ependymoma is associated with high morbidity ${ }^{45}$. Interestingly, there was no difference in resection rates between high and low-volume surgical centres. There is a trend to refer children to higher volume centres for surgery, but our data would not support this. However, this is based on a small retrospective analysis and should not change practice.

The protocol requested radiotherapy information including: baseline imaging used to determine gross tumour volume; copies of the radiotherapy plan and treatment chart; and copies of the simulator and machine verification films for quality assurance. However, there was no established process in place to facilitate this and hence full radiotherapy dosimetric data was not available for analysis. The trial was carried out during a time of transition from 2D simulator planning, to CT assisted 3D planning and the technical details reflect this. In the 20 years since the design of this study, intensity-modulated radiation therapy, volumetric modulated arc therapy, helical tomotherapy and proton beam therapy have become standard of care for ependymoma and the radiotherapy techniques in this trial, including the dose of 54 rather than $59.4 \mathrm{~Gy}$, do not reflect contemporary practice. 
This prospective clinical trial was designed prior to recent molecular discoveries; however, it provided an opportunity to apply retrospective molecular analyses to a well-defined cohort, allowing comparison with other studies ${ }^{46}$. The results of central histopathology review and DNA methylation classification aligned closely with one another, however half of the cohort did not undergo DNA methylation profiling. Future studies must mandate the provision of tissue for molecular analyses, because they have the potential to provide more objective tumour classification for trial stratification and biological discovery ${ }^{47}$.

The finding that $1 q$ gain was associated with poor outcome confirms previous reports ${ }^{23,25,26}$. In-line with ACNS0121 we assessed 1q gain across supratentorial and PF tumours and found it to only be associated with poorer outcomes in children with PF tumours ${ }^{42}$. The prevalence of $1 q$ gain in our cohort was higher than reported by others ${ }^{21,48,49}$. One possibility is that this is a result of inclusion of multiple methods of testing, detecting additional cases that may not have been identified by FISH alone. Alternatively, there may be a genuinely higher rate of 1q gain, but we were unable to identify any abnormality that would account for this.

hTERT mRNA expression was associated with poorer outcomes. However, this analysis was conducted in a small subgroup of the whole cohort. Notwithstanding this, our finding is consistent with the previous reports for ependymoma expressing $h T E R T^{23}$. We also confirmed previous findings limiting $h T E R T$ expression to PFA and ZFTA-fused ependymomas ${ }^{24,27}$.

Loss of H3K27me3 expression was associated with poorer OS, likely reflecting the strong association between loss of this marker and PFA tumours ${ }^{28,29}$. TNC expression and absence of H3K27me3 was limited to PFA ${ }^{20}$. Given that TNC is a marker for PFA, based on the other samples tested with no DNA 
methylation result, there was likely to be at least a further nine PFA tumours in our cohort, indicating that $70 \%$ of our cohort were PFA, consistent with it being the most common childhood ependymoma type. RELA protein has also been reported as an immunohistochemical marker for ZFTA-fused ependymoma ${ }^{27,50}$, however, we identified RELA expression across multiple subtypes, indicating that RELA expression alone is not suitable for identifying these tumours. A potential prognostic parameter is $C D K N 2 A / B$ which seems to be associated with particularly poor prognosis in retrospective studies ${ }^{40}$. Whilst we did not measure this it presents a further route of investigation for ZFTA-fused ependymomas.

The retrospective nature of the methylation analysis resulted in low numbers of each subtype, highlighting the challenges of obtaining sufficient tissue. When designing prospective molecularly stratified studies, estimates of cohort size must account for this. It is also clear that with the description of new molecular entities this stratification will become even more challenging ${ }^{18,37}$. In some settings obtaining methylation results in time for prompt stratification can be challenging or impossible, therefore it is important to continue to identify reliable but robust alternative markers for molecular groups.

\section{Conclusions}

We confirm the importance of GTR in childhood ependymoma and present evidence that amongst those with STR there is activity of VEC with a RR of $65.5 \%(95 \% \mathrm{Cl} 45.7-82.1)$ with acceptable toxicity profile. This supports a potential beneficial role for chemotherapy in children with intracranial ependymoma. However, as this study was not designed to measure changes in EFS and OS, further data is required to determine whether there is an associated survival impact. Further work is needed to establish whether this varies depending on the molecular tumour designation. We confirm that 
1q gain, hTERT expression and H3K27me3 loss are poor prognostic factors for intracranial ependymoma. Future trials must include prospective, molecularly stratified, approaches to better understand the clinical implications of recent molecular discoveries.

Acknowledgements: We acknowledge the CRUK clinical trials unit team including Andrew Raxworthy-Cooper for study support. TR acknowledges the support of 'Fighting Ependymoma'. RGG acknowledges the support of the Joseph Foote Trust (now part of the Brain Tumour Charity). 


\section{References}

1. Ritzmann TA, Rogers HA, Paine SML, et al. A retrospective analysis of recurrent pediatric ependymoma reveals extremely poor survival and ineffectiveness of current treatments across central nervous system locations and molecular subgroups. Pediatr Blood Cancer. 2020;67(9).

2. De B, Khakoo Y, Souweidane MM, et al. Patterns of relapse for children with localized intracranial ependymoma. J Neurooncol. 2018;138(2):435-445.

3. Jünger ST, Mynarek M, Wohlers I, et al. Improved risk-stratification for posterior fossa ependymoma of childhood considering clinical, histological and genetic features - A retrospective analysis of the HIT ependymoma trial cohort. Acta Neuropathol Commun. 2019;7(1).

4. Massimino M, Miceli R, Giangaspero F, et al. Final results of the second prospective AIEOP protocol for pediatric intracranial ependymoma. Neuro-Oncol. 2016;18(10):1451-1460.

5. Godfraind C, Kaczmarska JM, Kocak M, et al. Distinct disease-risk groups in pediatric supratentorial and posterior fossa ependymomas. Acta Neuropathol. 2012;124(2):247-257.

6. Garvin J, Selch MT, Holmes E, et al. Phase II Study of Pre-Irradiation Chemotherapy for Childhood Intracranial Ependymoma. Children's Cancer Group Protocol 9942: A Report From the Children's Oncology Group. Pediatr Blood Cancer. 2012;59(7):1183-1189.

7. Jaing T-H, Wang H-S, Tsay P-K, et al. Multivariate Analysis of Clinical Prognostic Factors in Children with Intracranial Ependymomas. J Neurooncol. 2004;68(3):255-261.

8. Paulino AC, Wen B-C, Buatti JM, et al. Intracranial ependymomas: an analysis of prognostic factors and patterns of failure. Am J Clin Oncol. 2002;25(2):117-122. 
9. Rudà R, Reifenberger G, Frappaz D, et al. EANO guidelines for the diagnosis and treatment of ependymal tumors. Neuro-Oncol. 2018;20(4):445-456.

10. Khatua S, Ramaswamy V, Bouffet E. Current therapy and the evolving molecular landscape of paediatric ependymoma. Eur J Cancer. 2017;70:34-41.

11. Duffner $\mathrm{P}$, Horowitz $\mathrm{M}$, Krischer J, et al. Postoperative chemotherapy and delayed radiation in children less than three years of age with malignant brain tumours. New Engl J Med. 1993;328(24):1725-1731.

12. Strother DR, Lafay-Cousin L, Boyett JM, et al. Benefit from prolonged dose-intensive chemotherapy for infants with malignant brain tumors is restricted to patients with ependymoma: a report of the Pediatric Oncology Group randomized controlled trial 9233/34. Neuro-Oncol. 2014;16(3):457-465.

13. Geyer JR, Sposto R, Jennings M, et al. Multiagent Chemotherapy and Deferred Radiotherapy in Infants With Malignant Brain Tumors : A Report From the Children 's Cancer Group. J Clin Oncol. 2005;23(30):9-11.

14. Grill J, le Deley MC, Gambarelli D, et al. Postoperative chemotherapy without irradiation for ependymoma in children under 5 years of age: a multicenter trial of the French Society of Pediatric Oncology. J Clin Oncol. 2001;19(5):1288-1296.

15. Grundy RG, Wilne S, Weston $\mathrm{CL}$, et al. Primary postoperative chemotherapy without radiotherapy for intracranial ependymoma in children: the UKCCSG/SIOP prospective study. Lancet Oncol. 2007;8(8):696-705.

16. Venkatramani R, Ji L, Lasky J, et al. Outcome of infants and young children with newly diagnosed ependymoma treated on the "head Start" III prospective clinical trial. J Neurooncol. 2013;113(2):285-291. 
17. Evans AE, Anderson JR, Lefkowitz-boudreaux IB. Adjuvant Chemotherapy of Childhood Posterior Fossa Ependymoma: Cranio-Spinal Irradiation With or Without Adjuvant CCN U, Vincristine, and Prednisone: A Children's Cancer Group Study. Med Pediatr Oncol. $1996 ; 27(1): 8-14$

18. Zheng T, Ghasemi DR, Okonechnikov K, et al. Cross-Species Genomics Reveals Oncogenic Dependencies in ZFTA/C11orf95 Fusion-Positive Supratentorial Ependymomas. Cancer Discov. 2021;11(9):2230-2247.

19. Louis DN, Perry A, Wesseling P, et al. The 2021 WHO Classification of Tumors of the Central Nervous System: a summary. Neurooncol. 2021;23(8):1231-1251.

20. Pajtler KW, Witt H, Sill M, et al. Molecular Classification of Ependymal Tumors across All CNS Compartments, Histopathological Grades, and Age Groups. Cancer Cell. 2015;27(5):728-743.

21. Kilday JP, Mitra B, Domerg C, et al. Copy number gain of $1 \mathrm{q} 25$ predicts poor progression-free survival for pediatric intracranial ependymomas and enables patient risk stratification. Clin Cancer Res. 2012;18(7):2001-2011.

22. Upadhyaya SA, Robinson GW, Onar-Thomas A, et al. Molecular grouping and outcomes of young children with newly diagnosed ependymoma treated on the multi-institutional SJYC07 trial. Neurooncol. 2019;21(10):1319-1330.

23. Gojo J, Lötsch D, Spiegl-Kreinecker S, et al. Telomerase activation in posterior fossa group A ependymomas is associated with dismal prognosis and chromosome 1q gain. Neurooncol. 2017;19(9):1183-1194.

24. Araki A, Chocholous M, Gojo J, et al. Chromosome 1q gain and tenascin-C expression are candidate markers to define different risk groups in pediatric posterior fossa ependymoma. Acta Neuropathol Commun. 2016;4(1):88. 
25. Tabori $\mathrm{U}$, Wong $\mathrm{V}, \mathrm{Ma} \mathrm{J}$, et al. Telomere maintenance and dysfunction predict recurrence in paediatric ependymoma. Br J Cancer. 2008;99(7):1129-1135.

26. Modena P, Buttarelli FR, Miceli R, et al. Predictors of outcome in an AIEOP series of childhood ependymomas: A multifactorial analysis. Neurooncol. 2012;14(11):1346-1356.

27. Andreiuolo F, le Teuff G, Bayar MA, et al. Integrating Tenascin-C protein expression and 1q25 copy number status in pediatric intracranial ependymoma prognostication: A new model for risk stratification. PLoS One. 2017;12(6).

28. Bayliss J, Mukherjee P, Lu C, et al. Lowered H3K27me3 and DNA hypomethylation define poorly prognostic pediatric posterior fossa ependymomas. Sci Trans/ Med. 2016;8(366):366ra161.

29. Panwalkar P, Clark J, Ramaswamy V, et al. Immunohistochemical analysis of H3K27me3 demonstrates global reduction in group-A childhood posterior fossa ependymoma and is a powerful predictor of outcome. Acta Neuropathol. 2017;134(5):705-714.

30. Li AM, Dunham C, Tabori U, et al. EZH2 expression is a prognostic factor in childhood intracranial ependymoma: A Canadian Pediatric Brain Tumor Consortium study. Cancer. 2015;121(9):1499-1507.

31. Gnekow AK. Recommendations of the brain tumor subcommittee for the reporting of trials. Med Pediatr Oncol. 1995;24(2):104-108.

32. Robertson PL, Zeltzer PM, Boyett JM, et al. Survival and prognostic factors following radiation therapy and chemotherapy for ependymomas in children: a report of the Children's Cancer Group. J Neurosurg. 1998;88(4):695-703. 
33. Bouffet E, Perilongo G, Canete A, Massimino M. Intracranial ependymomas in children: A critical review of prognostic factors and a plea for cooperation. Med Pediatr Oncol. 1998;30(6):319-329.

34. Bouffet E, Foreman N. Chemotherapy for Intracranial ependymomas. Child's Nerv Sys. 1999;15:563-570.

35. Machin D, Campbell M, Tan S, Tan S. Sample Sizes for Clinical Studies: Phase II Trials. 3rd ed. Wiley-Blackwell; 2009.

36. Clopper CJ, Pearson ES. The use of confidence or fiducial limits illustrated in the case of the binomial. Biometrika. 1934;26(4):404-413.

37. Sievers P, Henneken SC, Blume C, et al. Recurrent fusions in PLAGL1 define a distinct subset of pediatric-type supratentorial neuroepithelial tumors. Acta Neuropathol. 2021;142(5).

38. Ellison DW, Aldape KD, Capper D, et al. CIMPACT-NOW update 7: advancing the molecular classification of ependymal tumors. Brain Pathol. 2020;30(5):863-866.

39. Pajtler KW, Wen J, Sill M, et al. Molecular heterogeneity and CXorf67 alterations in posterior fossa group A (PFA) ependymomas. Acta Neuropathol. 2018;136(2):211-226.

40. Jünger $\mathrm{S}$, Andreiuolo $\mathrm{F}$, Mynarek $\mathrm{M}$, et. al. CDKN2A deletion in supratentorial ependymoma with RELA alteration indicates a dismal prognosis: a retrospective analysis of the HIT ependymoma trial cohort. Acta Neuropathol. 2020;140:405-407.

41. Smith A, Onar-Thomas A, Ellison D, et al. EPEN-54. ACNS0831, PHASE III RANDOMIZED TRIAL OF POST-RADIATION CHEMOTHERAPY IN PATIENTS WITH NEWLY DIAGNOSED EPENDYMOMA AGES 1 TO 21 YEARS. Neurooncol. 2020;22(Supplement_3). 
42. Merchant TE, Bendel AE, Sabin ND, et al. Conformal Radiation Therapy for Pediatric Ependymoma, Chemotherapy for Incompletely Resected Ependymoma, and Observation for Completely Resected, Supratentorial Ependymoma. J Clin Oncol. 2019;37(12):974-983

43. Grist JT, Withey S, MacPherson L, et al. Distinguishing between paediatric brain tumour types using multi-parametric magnetic resonance imaging and machine learning: A multi-site study. Neurolmage Clin. 2020;25;102172.

44. Millward CP, Mallucci C, Jaspan T, et al. Assessing 'second-look' tumour resectability in childhood posterior fossa ependymoma - a centralised review panel and staging tool for future studies. Childs Nerv Sys. 2016;32(11):2189-2196.

45. Sanford RA, Merchant TE, Zwienenberg-Lee M, et. al. Advances in surgical techniques for resection of childhood cerebellopontine angle ependymomas are key to survival. Childs Nerv Sys. 2009;25(10):1229-1240.

46. Massimino M, Barretta F, Modena $\mathrm{P}$, et al. The AIEOP 2nd series of children and adolescents intracranial Ependymoma. An integrated molecular and clinical characterization with a longterm follow-up. Neurooncol. 2021;23(5):848-857.

47. Ellison DW, Kocak M, Figarella-Branger D, et al. Histopathological grading of pediatric ependymoma: reproducibility and clinical relevance in European trial cohorts. J Negat Results Biomed. 2011;10(1):7.

48. Carter M, Nicholson J, Ross F, et al. Genetic abnormalities detected in ependymomas by comparative genomic hybridisation. Br J Cancer. 2002;86(6):929-939.

49. Mendrzyk F, Korshunov A, Benner A, et al. Identification of gains on 1q and epidermal growth factor receptor overexpression as independent prognostic markers in intracranial ependymoma. Clin Cancer Res. 2006;12(16):2070-2079. 
50. Parker M, Mohankumar KM, Punchihewa C, et al. C11orf95-RELA fusions drive oncogenic NFKB signalling in ependymoma. Nature. 2014;506(7489):451-455. 
Figure 1: (A) Protocol defined flow through study. (B) Actual flow. 41 had STR and 33 GTR. Of those with STR, 29 received chemotherapy, 10 received radiotherapy and two had no further therapy. 32/33 with GTR received radiotherapy and one had no further treatment. Grey boxes indicate protocol violations. (C) Chemotherapy outline, further detail in Supplementary Material 1. (D) Overall and (E) Event Free survival for the entire cohort $(n=74)$.

Figure 2: Kaplan-Meier estimators. (A) STR associated with poorer EFS than GTR. (B) Grade 3 associated with worse OS than grade 2 tumours. 1q gain had worse EFS (C) and OS (D). H3K27me3 loss had worse OS (E). hTERT expression associated with worse EFS than no expression (F). EFS (G) and OS $(\mathrm{H})$ stratified by methylation group did not differ significantly. However, patterns of survival reflect previously published data.

Figure 3: (A) Summary of responses to chemotherapy as assessed by MR imaging after 2- and 4cycles and best overall response. (B) Swimmers plot of all participants who received chemotherapy and centrally assessed response assessment findings at cycles two and four. Colours relate to best response achieved, black symbols represent response obtained at each assessment. Line length reflects number of cycles each participant received. 
Figure 4: (A) Subdivision of methylation results. 34 participants had ependymoma assessed by methylation array. 17 were PFA, 10 ZFTA-fusion, 5 PFB, one YAP and one ST_PLAGL1. Of the remaining 40, 12 had a low classifier score and 28 had no tissue. (B) Outcomes of molecular and immunohistochemical analyses stratified by methylation classification. 1q gain seen only in PFA and ZFTA-fusion and hTERT only in PFA and ZFTA-fusion. In PF tumours, Tenascin-C and H3K27me3 only identified in PFA and PFB respectively. ReLA IHC was not a good marker for ZFTA tumours; five cases of PFA demonstrated RELA positivity. No classification includes tumours with no methylation result for any reason. 


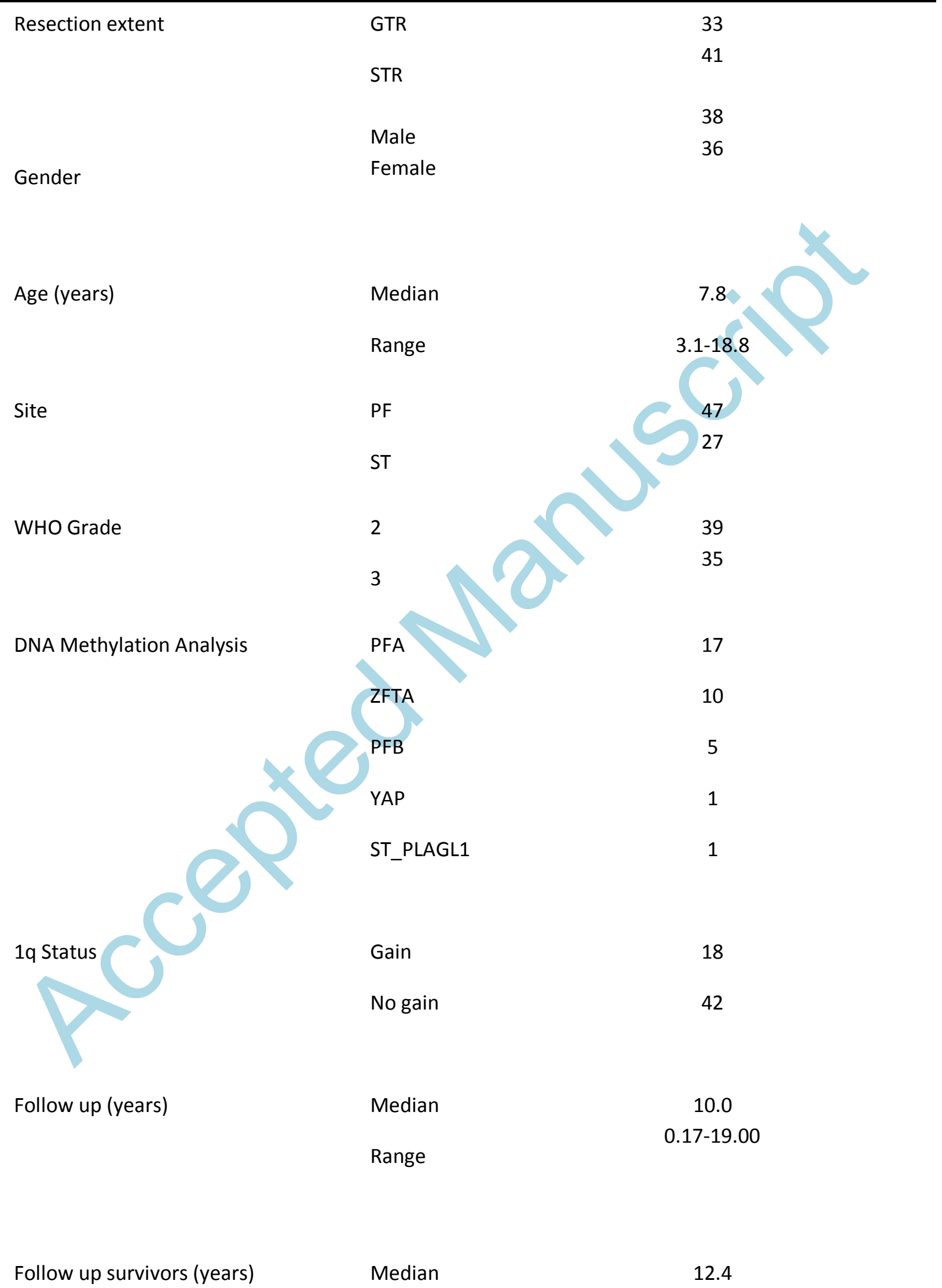


Range

Event Free Survival (\%)

$95 \% \mathrm{Cl}$

10-year

Overall Survival (\%)

$95 \% \mathrm{Cl}$

10-year
46.7

36.5-59.7

1.16-19.00

49.5

39.3-62.4

\section{3}

59.4-80.9

60.5

50.1-73.1

Table 1: Summary of key cohort characteristics, overall and event free survival times. Cl: Confidence Interval, GTR: Gross Total Resection, STR: Subtotal Resection, PF: Posterior fossa, ST: Supratentorial. PLAGL1: Neuroepithelial tumour, PLAGL1fusion positive. 


\begin{tabular}{|c|c|c|c|c|c|c|c|c|c|c|}
\hline & \multirow[b]{2}{*}{$\mathbf{n}$} & \multicolumn{2}{|c|}{ EFS (95\% Cl) } & \multirow{2}{*}{$\begin{array}{c}\text { Hazard } \\
\text { Ratio } \\
(95 \% \mathrm{Cl})\end{array}$} & \multirow[b]{2}{*}{$\mathbf{P}$} & \multicolumn{2}{|c|}{ OS $(95 \% \mathrm{Cl})$} & \multirow{2}{*}{$\begin{array}{c}\text { Hazard } \\
\text { Ratio } \\
(95 \% \mathrm{Cl})\end{array}$} & \multirow[b]{2}{*}{$\mathbf{P}$} \\
\hline & & & 5 Year & 10 Year & & & 5 Year & 10 Year & & \\
\hline \multicolumn{2}{|c|}{ Whole Cohort } & 74 & $\begin{array}{c}49.5 \\
(39.3- \\
62.4) \\
\end{array}$ & $\begin{array}{c}46.7 \\
(36.5- \\
59.7) \\
\end{array}$ & & & $\begin{array}{c}69.3 \\
(59.4- \\
80.9) \\
\end{array}$ & $\begin{array}{c}60.5 \\
(50.1- \\
73.1) \\
\end{array}$ & & \\
\hline & & & & & & & & & & \\
\hline \multirow{2}{*}{ Resection } & GTR & 33 & $\begin{array}{c}69.1 \\
(54.9- \\
87.1) \\
\end{array}$ & $\begin{array}{r}62.9 \\
(48.2- \\
82.0) \\
\end{array}$ & 1 & \multirow{2}{*}{0.003} & $\begin{array}{r}81.3 \\
(68.9- \\
96.0) \\
\end{array}$ & $\begin{array}{r}68.0 \\
(53.4- \\
86.6) \\
\end{array}$ & 1 & \multirow{2}{*}{0.071} \\
\hline & STR & 41 & $\begin{array}{c}33.8 \\
(22.0- \\
52.0) \\
\end{array}$ & $\begin{array}{r}33.8 \\
(22.0- \\
52.0) \\
\end{array}$ & $\begin{array}{c}2.6(1.4- \\
5.1)\end{array}$ & & $\begin{array}{c}59.5 \\
(45.9- \\
77.0) \\
\end{array}$ & $\begin{array}{c}54.3 \\
(40.7- \\
72.4) \\
\end{array}$ & $\begin{array}{c}2.0 \\
(0.93- \\
4.2) \\
\end{array}$ & \\
\hline \multirow{2}{*}{$\begin{array}{l}\text { WHO } \\
\text { Grade }\end{array}$} & 2 & 39 & $\begin{array}{c}59.0 \\
(45.4- \\
76.6) \\
\end{array}$ & $\begin{array}{c}56.4 \\
(42.8- \\
74.3) \\
\end{array}$ & 1 & \multirow{2}{*}{0.061} & $\begin{array}{c}84.2 \\
(73.3- \\
96.6)\end{array}$ & $\begin{array}{c}78.8 \\
(66.7- \\
93.0) \\
\end{array}$ & & \multirow{2}{*}{0.005} \\
\hline & 3 & 35 & $\begin{array}{c}38.7 \\
(25.2- \\
59.2) \\
\end{array}$ & $\begin{array}{r}35.4 \\
(22.4- \\
56.1) \\
\end{array}$ & $\begin{array}{c}1.8 \\
(0.96- \\
3.3) \\
\end{array}$ & & $\begin{array}{c}52.2 \\
(37.7- \\
72.3) \\
\end{array}$ & $\begin{array}{c}39.9 \\
(26.2- \\
60.7)\end{array}$ & $\begin{array}{c}2.8(1.3- \\
5.8)\end{array}$ & \\
\hline & & & & & & & $x^{2}$ & & & \\
\hline \multirow{2}{*}{ Location } & PF & 47 & $\begin{array}{c}55.3 \\
(42.8- \\
71.5)\end{array}$ & $\begin{array}{c}51.1 \\
(38.6- \\
67.6)\end{array}$ & 1 & & $\begin{array}{c}69.6 \\
(57.5- \\
84.3)\end{array}$ & $\begin{array}{c}62.9 \\
(50.3- \\
78.6)\end{array}$ & 1 & \multirow{2}{*}{0.585} \\
\hline & ST & 27 & $\begin{array}{c}38.8 \\
(23.8- \\
63.3) \\
\end{array}$ & $\begin{array}{c}38.8 \\
(23.8- \\
63.3) \\
\end{array}$ & $\begin{array}{c}1.5 \\
(0.82- \\
2.8)\end{array}$ & & $\begin{array}{c}68.5 \\
(52.5- \\
89.3) \\
\end{array}$ & $\begin{array}{c}55.6 \\
(39.0- \\
79.3) \\
\end{array}$ & $\begin{array}{c}1.2 \\
(0.59- \\
2.5) \\
\end{array}$ & \\
\hline & & & & & & & & & & \\
\hline \multirow[t]{2}{*}{ 1q Status } & Gain & 18 & $\begin{array}{c}33.3 \\
(17.3- \\
64.1) \\
\end{array}$ & $\begin{array}{c}22.2 \\
(9.4- \\
52.7)\end{array}$ & 1 & \multirow{2}{*}{0.003} & $\begin{array}{c}55.6 \\
(36.8- \\
84.0) \\
\end{array}$ & $\begin{array}{c}44.4 \\
(26.5- \\
74.5) \\
\end{array}$ & 1 & \multirow{2}{*}{0.042} \\
\hline & No Gain & 42 & $\begin{array}{c}59.0 \\
(45.7- \\
76.1)\end{array}$ & $\begin{array}{c}59.0 \\
(45.7- \\
76.1) \\
\end{array}$ & $\begin{array}{c}0.37 \\
(0.19- \\
0.74) \\
\end{array}$ & & $\begin{array}{c}75.5 \\
(64.4- \\
90.0) \\
\end{array}$ & $\begin{array}{c}68.0 \\
(55.0- \\
84.0) \\
\end{array}$ & $\begin{array}{c}0.45 \\
(0.21- \\
0.99) \\
\end{array}$ & \\
\hline & & & 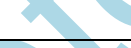 & & & & & & & \\
\hline \multirow[t]{2}{*}{ H3К27me3 } & Positive & 22 & $\begin{array}{c}67.6 \\
(50.5- \\
90.6)\end{array}$ & $\begin{array}{c}62.8 \\
(45.3- \\
80.7) \\
\end{array}$ & 1 & \multirow{2}{*}{0.165} & $\begin{array}{c}90.2 \\
(78.2- \\
100) \\
\end{array}$ & $\begin{array}{c}85.2 \\
(71.1- \\
100) \\
\end{array}$ & 1 & \multirow{2}{*}{0.003} \\
\hline & Negative & 32 & $\begin{array}{c}40.6 \\
(26.7- \\
61.8) \\
\end{array}$ & $\begin{array}{c}37.5 \\
(24.0- \\
58.7) \\
\end{array}$ & $\begin{array}{c}1.72 \\
(0.77- \\
3.9) \\
\end{array}$ & & $\begin{array}{c}46.9 \\
(32.4- \\
67.8) \\
\end{array}$ & $\begin{array}{c}40.6 \\
(26.7- \\
61.8) \\
\end{array}$ & $\begin{array}{c}4.6(1.5- \\
13.2)\end{array}$ & \\
\hline & 1 & & & & & & & & & \\
\hline \multirow[t]{2}{*}{ hTERT } & Negative & 6 & $\begin{array}{c}83.3 \\
(58.3- \\
100)\end{array}$ & $\begin{array}{c}66.7 \\
(37.9- \\
100) \\
\end{array}$ & 1 & \multirow{2}{*}{0.014} & 100 & $\begin{array}{c}83.3 \\
(58.3- \\
100)\end{array}$ & 1 & \multirow{2}{*}{0.092} \\
\hline & Positive & 10 & $\begin{array}{l}20.0 \\
(5.8- \\
69.1)\end{array}$ & $\begin{array}{l}20.0 \\
(5.8- \\
69.1)\end{array}$ & $\begin{array}{c}5.8(1.2- \\
28)\end{array}$ & & $\begin{array}{c}50.0 \\
(26.9- \\
92.9) \\
\end{array}$ & $\begin{array}{c}40.0 \\
(18.7- \\
85.5)\end{array}$ & $\begin{array}{c}5.2 \\
(0.62- \\
43)\end{array}$ & \\
\hline
\end{tabular}

Table 2: Univariate survival analyses for key clinical and molecular features. Whilst clinical features were defined for analysis prospectively, the additional molecular analyses were post hoc. GTR: Gross Total Resection, STR: Subtotal resection, PF: Posterior Fossa, ST: Supratentorial, Cl: Confidence Interval, EFS: Event Free Survival, OS: Overall Survival. 
Figure 1

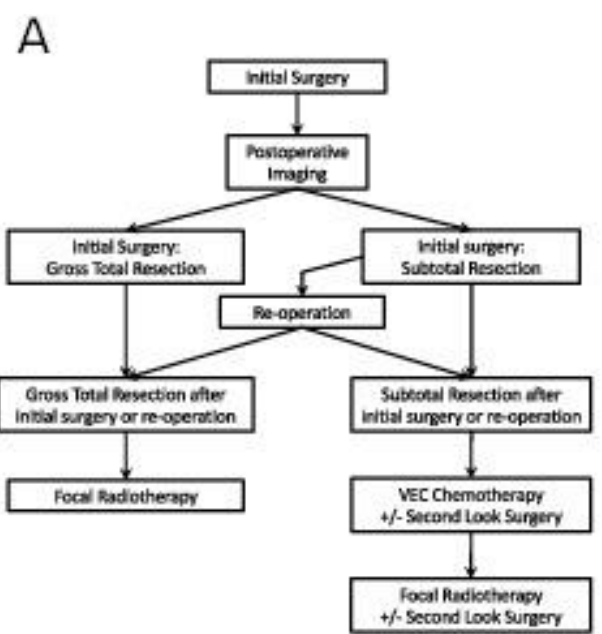

B
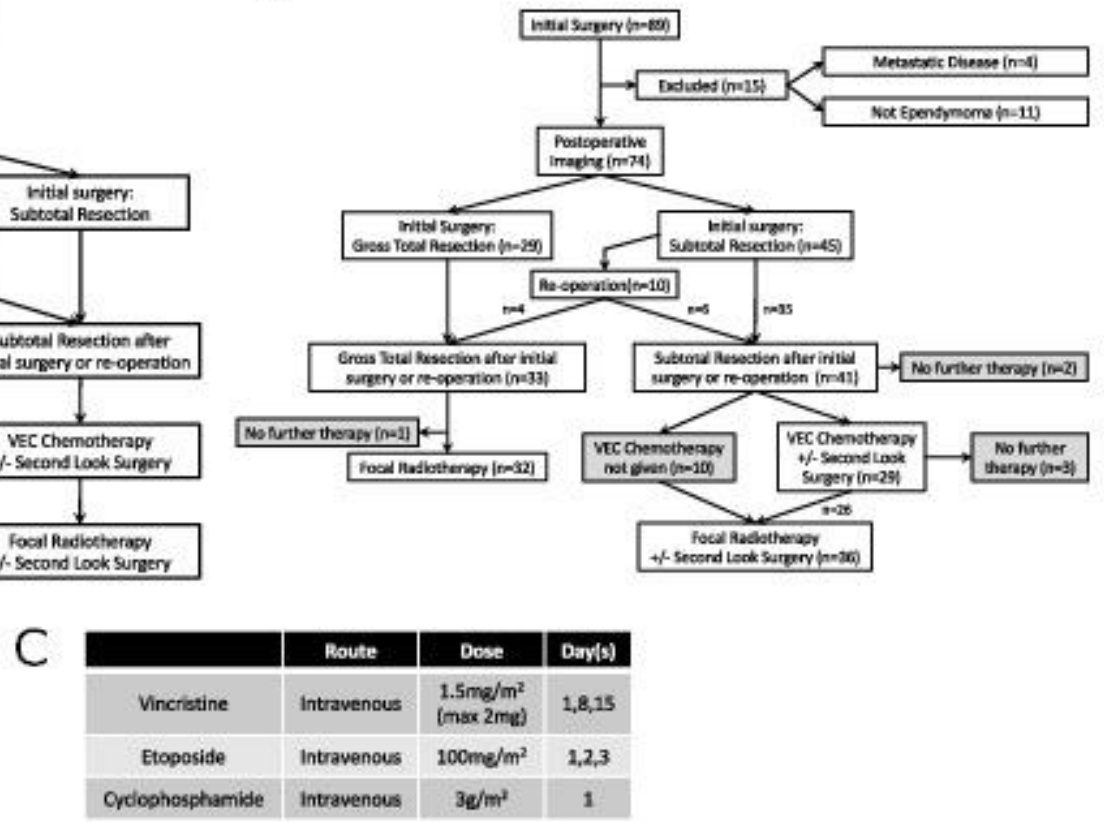

D
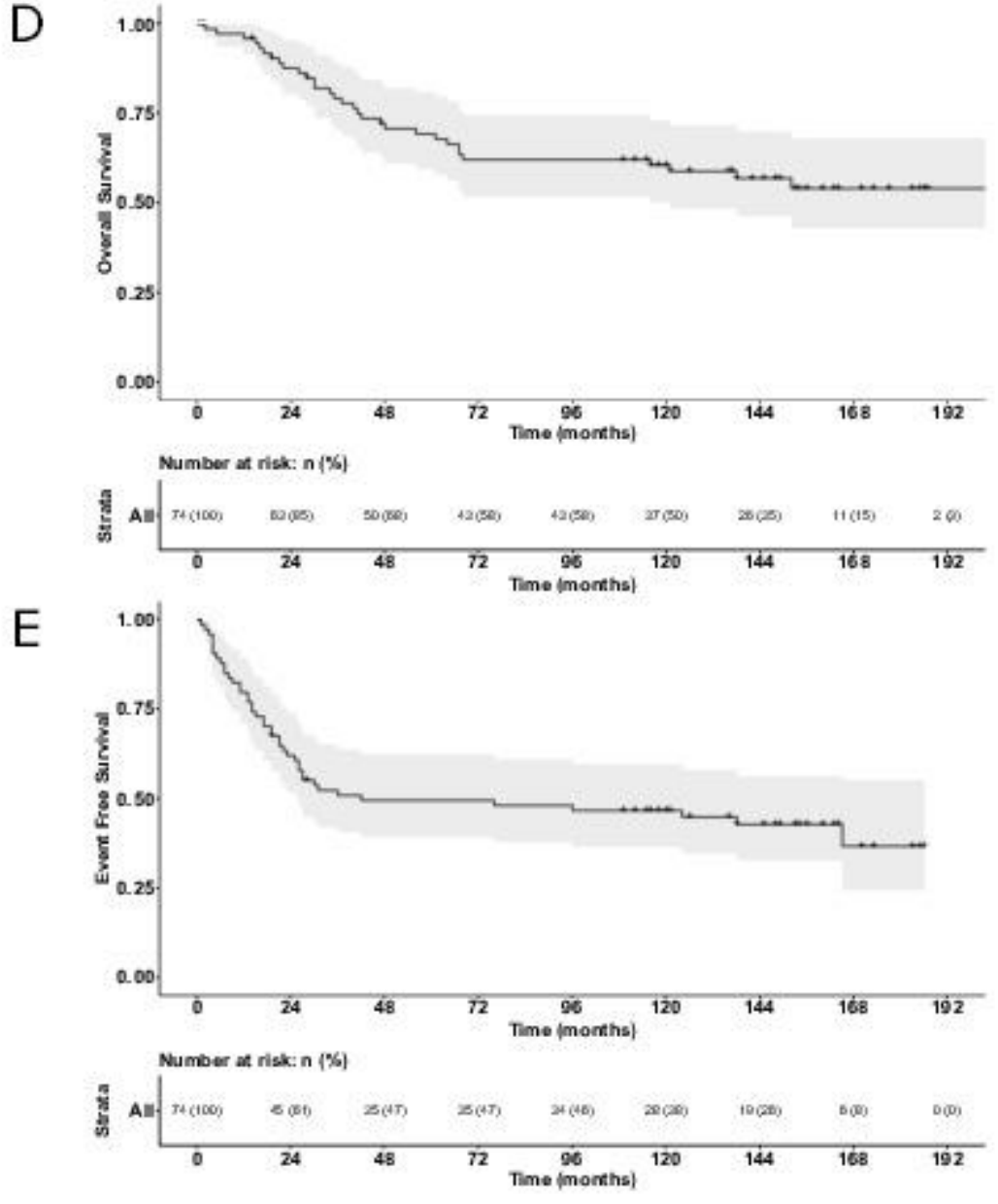
Figure 2
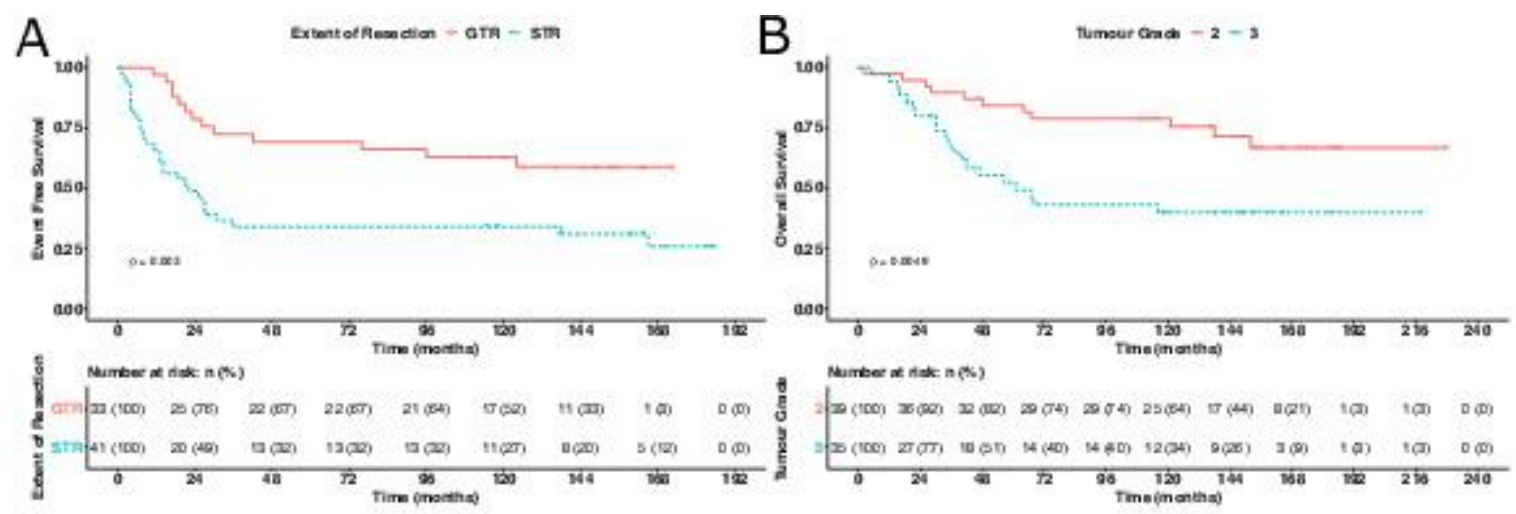

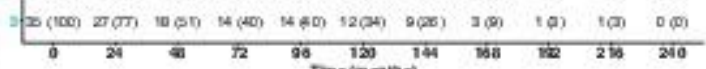

C

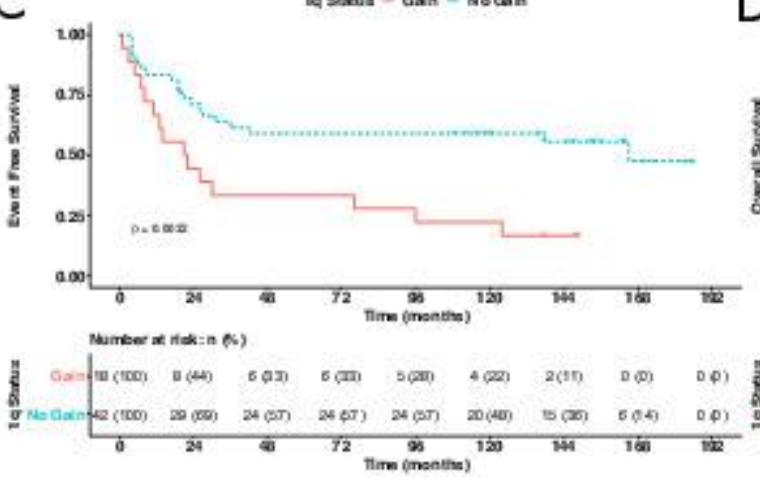

E

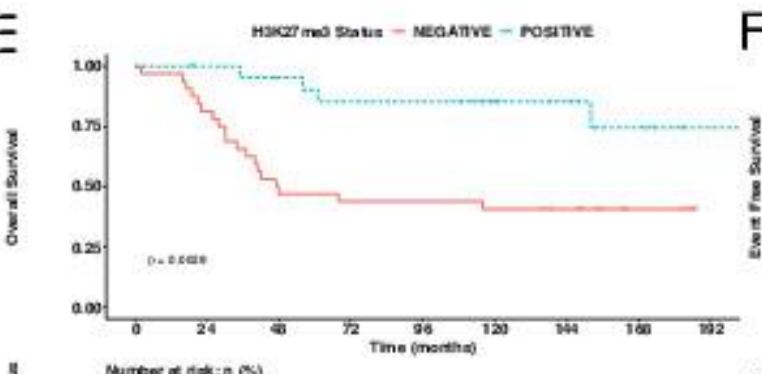

F

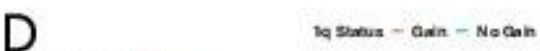

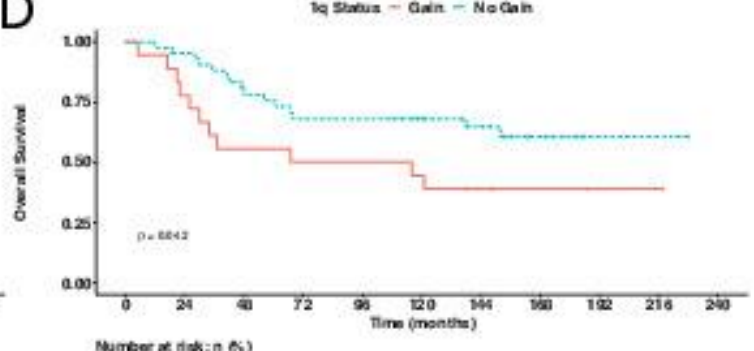

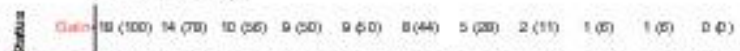

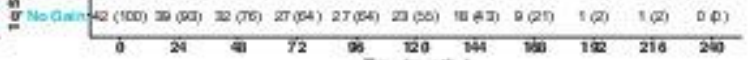

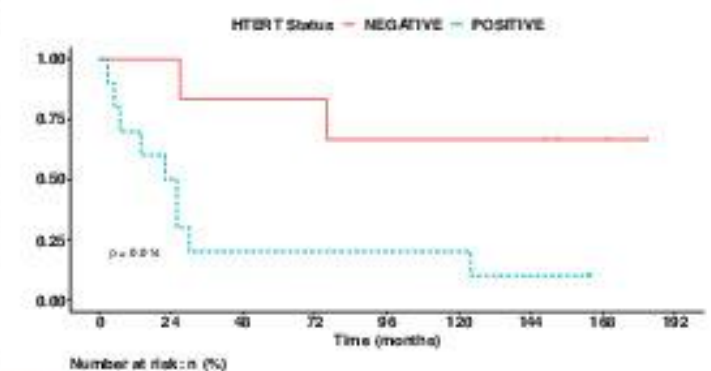

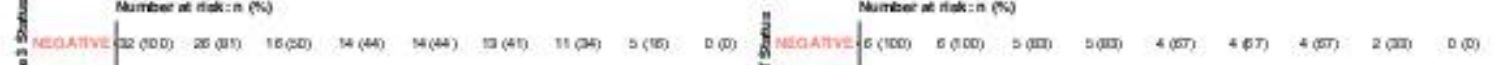

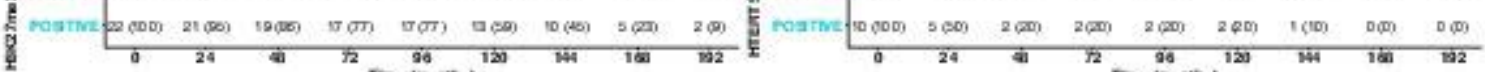
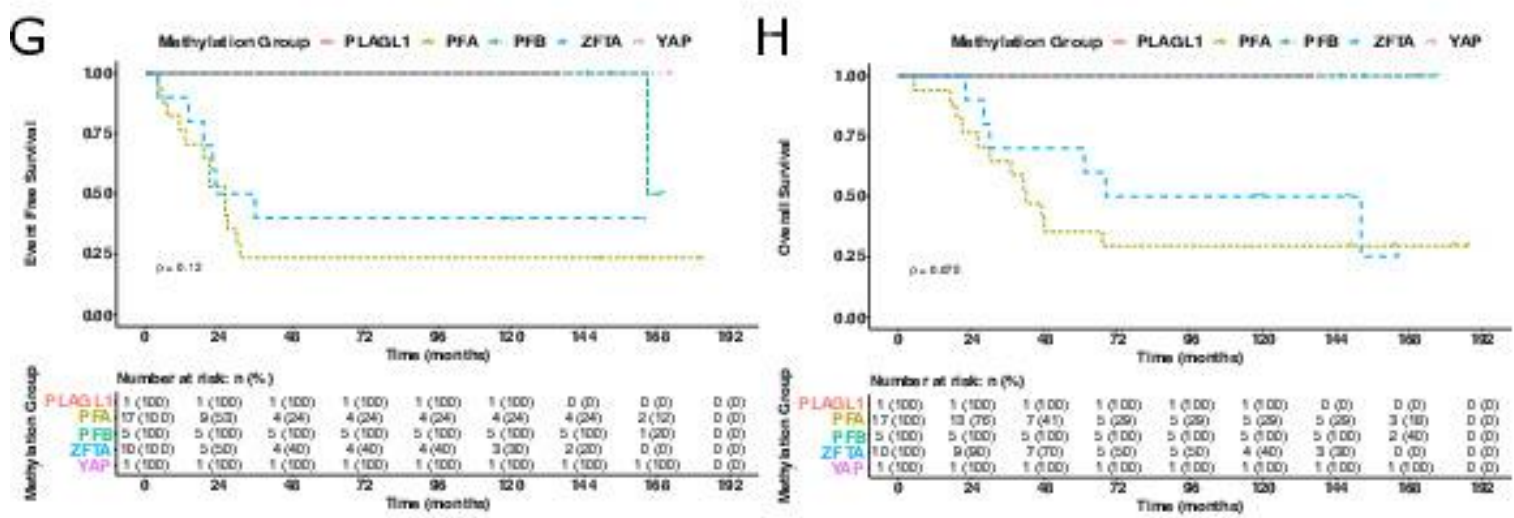
Figure 3

A

\begin{tabular}{cccc}
\hline & Cycle 2 Response & Cycle 4 response & Best Response \\
\hline CR & 6 & 5 & 9 \\
\hline PR & 8 & 8 & 10 \\
\hline OR & 2 & 0 & 0 \\
\hline SD & 6 & 3 & 4 \\
\hline PD & 6 & 6 & 6 \\
\hline CR+PR (\%) & $14 / 28(50.0)$ & $13 / 22(59.1)$ & $19 / 29(65.5)$ \\
95\% Cl & $30.7-69.4$ & $36.4-79.3$ & $45.7-82.1$ \\
\hline
\end{tabular}

B

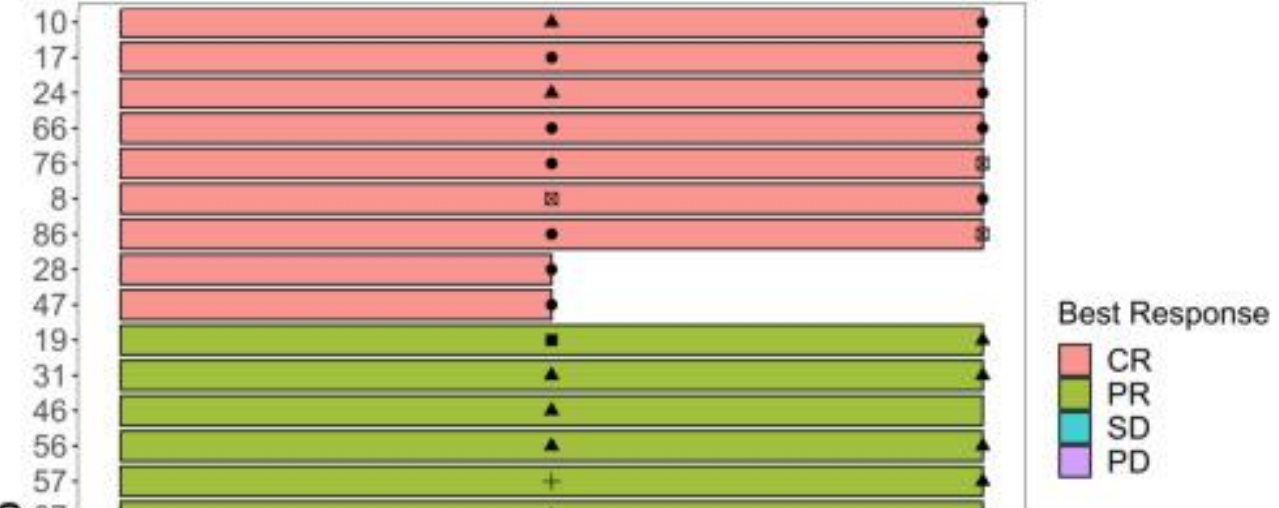

Cycle 2 or 4 Response

- CR

- PR

- OR

+ SD

\& $\mathrm{PD}$ 
Figure 4

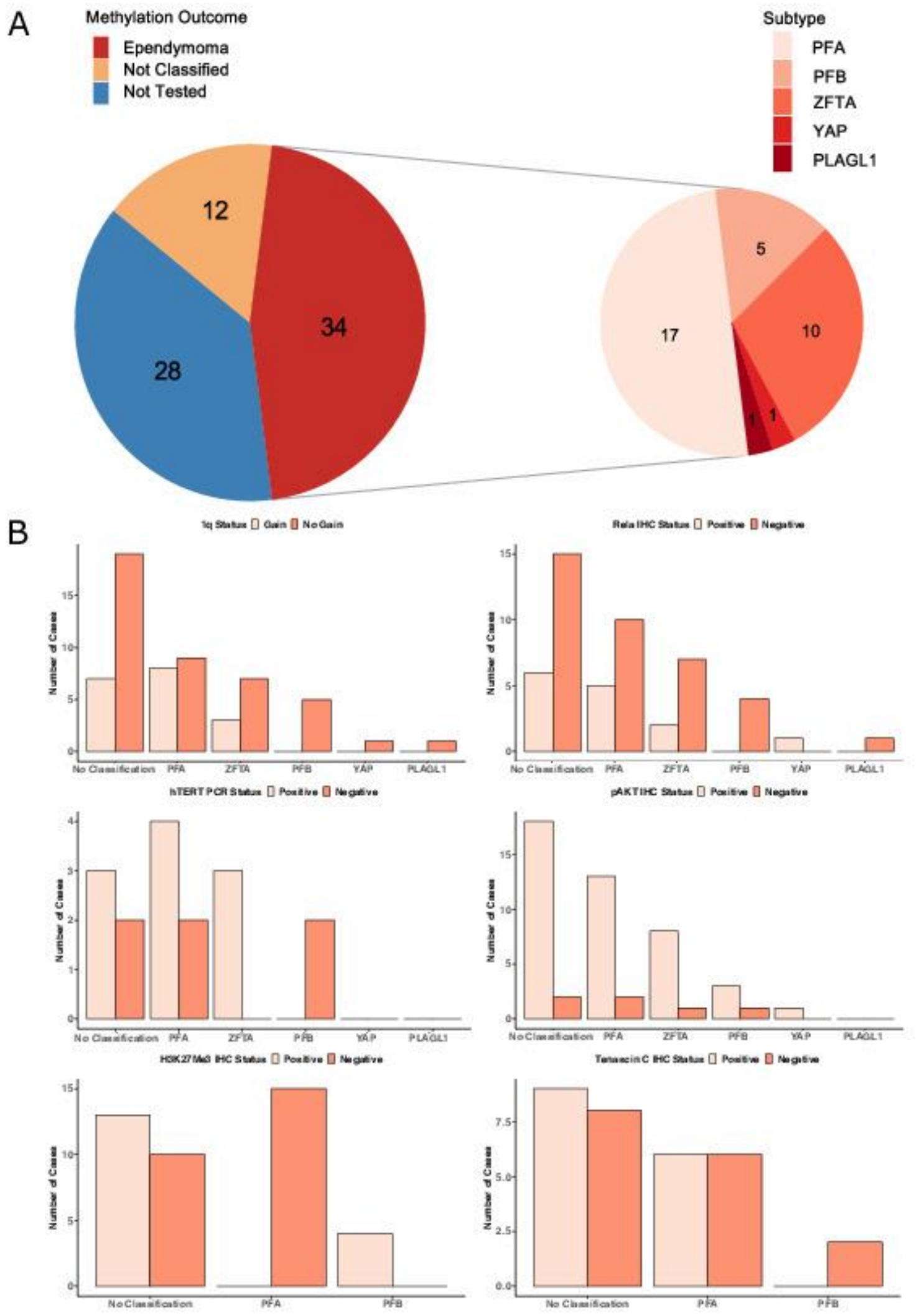

